\title{
A search for diffuse radio emission in the relaxed, cool-core galaxy clusters A1068, A1413, A1650, A1835, A2029, and Ophiuchus
}

\author{
F. Govoni ${ }^{1}$, M. Murgia ${ }^{1,2}$, M. Markevitch ${ }^{3}$, L. Feretti ${ }^{2}$, G. Giovannini ${ }^{2,4}$, G. B. Taylor ${ }^{5, \star}$, and E. Carretti ${ }^{2}$ \\ 1 INAF - Osservatorio Astronomico di Cagliari, Poggio dei Pini, Strada 54, 09012 Capoterra (CA), Italy \\ e-mail: fgovoni@ca.astro.it \\ 2 INAF - Istituto di Radioastronomia, via Gobetti 101, 40129 Bologna, Italy \\ Harvard-Smithsonian Center for Astrophysics, 60 Garden Street, Cambridge, MA 02138, USA \\ 4 Dipartimento di Astronomia, Univ. Bologna, via Ranzani 1, 40127 Bologna, Italy \\ 5 University of New Mexico, MSC 07 4220, Albuquerque, NM 87131, USA
}

Received 17 October 2008 / Accepted 28 January 2009

\section{ABSTRACT}

\begin{abstract}
Aims. We analyze sensitive, high-dynamic-range, observations to search for extended, diffuse, radio emission in relaxed and coolcore galaxy clusters.

Methods. We performed deep 1.4 GHz Very Large Array observations, of A1068, A1413, A1650, A1835, A2029, and complemented our dataset with archival observations of Ophiuchus.

Results. We find that, in the central regions of A1835, A2029, and Ophiuchus, the dominant radio galaxy is surrounded by diffuse low-brightness radio emission that takes the form of a mini-halo. We detect no diffuse emission in A1650, at a surface brightness level of the other mini-halos. We find low significance indications of diffuse emission in A1068 and A1413, although to be classified as mini-halos they would require further investigation, possibly with data of higher signal-to-noise ratio. In the Appendix, we report on the serendipitous detection of a giant radio galaxy with a total spatial extension of $\sim 1.6 \mathrm{Mpc}$.
\end{abstract}

Key words. galaxies: clusters: general - radio continuum: galaxies

\section{Introduction}

There is now firm evidence that the intra-cluster medium (ICM) consists of a mixture of hot plasma, magnetic fields, and relativistic particles. While the baryonic content of the galaxy clusters is dominated by the hot $(T \simeq 2-10 \mathrm{keV})$ intergalactic gas, whose thermal emission is observed in X-rays, a fraction of clusters also exhibit megaparsec-scale radio halos (see e.g., Feretti \& Giovannini 2008; Ferrari et al. 2008, and references therein for reviews). Radio halos are diffuse, low-surfacebrightness $\left(\simeq 10^{-6} \mathrm{Jy} / \operatorname{arcsec}^{2}\right.$ at $\left.1.4 \mathrm{GHz}\right)$, steep-spectrum ${ }^{1}(\alpha>$ 1) sources, permeating the central regions of clusters, produced by synchrotron radiation of relativistic electrons with energies of $\simeq 10 \mathrm{GeV}$ in magnetic fields with $B \simeq 0.5-1 \mu \mathrm{G}$. Radio halos represent the strongest evidence of large-scale magnetic fields and relativistic particles throughout the intra-cluster medium.

Radio halos are typically found in clusters that display significant evidence for an ongoing merger (e.g., Buote 2001; Govoni et al. 2004). Recent cluster mergers were proposed to play an important role in the reacceleration of the radio-emitting relativistic particles, thus providing the energy to these extended sources (e.g., Brunetti et al. 2001; Petrosian 2001).

To date about 30 radio halos are known (e.g., Giovannini \& Feretti 2000; Bacchi et al. 2003; Govoni et al. 2001; Venturi et al. 2007, 2008; Giovannini et al. in preparation). Because of their extremely low surface brightness and large angular extent $\left(>10^{\prime}\right.$ at a redshift $\left.z \leq 0.1\right)$ radio halos are most appropriately

* Greg Taylor is also an Adjunct Astronomer at the National Radio Astronomy Observatory.

${ }^{1} \mathrm{~S}(v) \propto v^{-\alpha}$. studied at low spatial resolution. Several radio halos were detected by Giovannini et al. (1999) in the NRAO VLA Sky Survey (NVSS; Condon et al. 1998) and by Kempner \& Sarazin (2001) in the Westerbork Northern Sky Survey (WENSS; Rengelink et al. 1997), where the relatively large beam of these surveys provide of the necessary sensitivity to large-scale emission in identifying these elusive sources.

A major merger event is expected to disrupt cooling cores and create disturbances that are readily visible in an X-ray image of the cluster. Therefore, the merger scenario predicts the absence of large-scale radio halos in symmetric cooling-core clusters. However, a few cooling-core clusters exhibit signs of diffuse synchrotron emission that extends far from the dominant radio galaxy at the cluster center, forming what is referred to as a mini-halo. These diffuse radio sources are extended on a moderate scale (typically $\simeq 500 \mathrm{kpc}$ ) and, in common with large-scale halos, have a steep spectrum and a very low surface brightness.

Because of a combination of small angular size and the strong radio emission of the central radio galaxy, the detection of a mini-halo requires data of a much higher dynamic range and resolution than those in available surveys, and this complicates their detection. As a consequence, our current observational knowledge on mini-halos is limited to only a handful of clusters (e.g., Perseus: Burns et al. 1992; A2390: Bacchi et al. 2003; RXJ1347.5-1145: Gitti et al. 2007), and their origin and physical properties are still poorly known. The study of radio emission from the center of cooling-core clusters is of large importance not only in understanding the feedback mechanism involved in the energy transfer between the AGN and the ambient medium (e.g., McNamara \& Nulsen 2007) but also in the 
formation process of the non-thermal mini-halos. The energy released by the central AGN may also play a role in the formation of these extended structures (e.g. Fujita et al. 2007).

On the other hand, the radiative lifetime of the relativistic electrons in mini-halos is of the order of $\simeq 10^{7}-10^{8} \mathrm{yr}$, much shorter than the time necessary for them to diffuse from the central radio galaxy to the mini-halo periphery. Thus, relativistic electrons must be reaccelerated and/or injected in-situ with high efficiency in mini-halos. Gitti et al. (2002) suggested that the mini-halo emission is due to a relic population of relativistic electrons reaccelerated by MHD turbulence via Fermi-like processes, the necessary energetics being supplied by the cooling flow. In support of mini-halo emission being triggered by the central cooling flow, Gitti et al. (2004) found a trend between the radio power of mini-halos and the cooling flow power. Although mini-halos are usually found in cooling-core clusters with no evidence of major mergers, signatures of minor-merging activities and gas-sloshing mechanisms in clusters containing minihalos (e.g., Gitti et al. 2007; Mazzotta \& Giacintucci 2008) have been revealed, suggesting that turbulence related to minor mergers could also play a role in the electron acceleration.

Alternatively, Pfrommer \& Enßlin (2004) proposed that relativistic electrons in mini-halos are of secondary origin and thus continuously produced by the interaction of cosmic ray protons with the ambient, thermal protons.

Cassano et al. (2008) found that the synchrotron emissivity (energy per unit volume, per unit time, per unit frequency) of mini-halos is about a factor of 50 higher than that of radio halos. In the framework of the particle re-acceleration scenario, they suggested that, an extra amount of relativistic electrons would be necessary to explain the higher radio emissivity of mini-halos. These electrons could be provided by the central radio galaxy or be of secondary origin.

To search for new extended diffuse radio emission in relaxed and cool-core galaxy clusters, we performed deep observations of A1068, A1413, A1650, A1835, and A2029, carried out with the Very Large Array at $1.4 \mathrm{GHz}$, and complemented our data set with a VLA archival observation of Ophiuchus. Here, we present the new mini-halos that we identified in these data. In Murgia et al. (submitted, hereafter Paper II), we quantitatively investigate the radio properties of these new sources and compare them with the radio properties of a statistically significant sample of mini-halos and halos already known in the literature, for which high quality VLA radio images at $1.4 \mathrm{GHz}$ are available.

The radio observations and data reduction are described in Sect. 2. For each cluster, in Sect. 3, we investigate the possible presence of a central mini-halo. In Sect. 4, we discuss the interplay between the mini-halos and the cluster X-ray emission. In Sect. 5, we analyze a possible connection between the central $\mathrm{cD}$ galaxy and the surrounding mini-halo. Finally, our conclusions are presented in Sect. 6.

Throughout this paper, we assume a $\Lambda$ CDM cosmology with $H_{0}=71 \mathrm{~km} \mathrm{~s}^{-1} \mathrm{Mpc}^{-1}, \Omega_{\mathrm{m}}=0.27$, and $\Omega_{\Lambda}=0.73$.

\section{VLA observations and data reduction}

To investigate the presence of diffuse, extended, radio emission in relaxed systems, we analyzed new and archival VLA data of cooling-core clusters. The list of the clusters is reported in Table 1, while the details of the observations are described in Table 2 .

We selected a small sample of 5 relaxed galaxy clusters: A1068, A1413, A1650, A1835, and A2029. They were selected
Table 1. List of the relaxed clusters analyzed in this work.

\begin{tabular}{cccc}
\hline \hline Cluster & $z$ & $\mathrm{kpc} /{ }^{\prime \prime}$ & $\begin{array}{c}D_{\mathrm{L}} \\
\mathrm{Mpc}\end{array}$ \\
\hline $\mathrm{A} 1068$ & 0.1375 & 2.40 & 641.45 \\
$\mathrm{~A} 1413$ & 0.1427 & 2.48 & 667.98 \\
$\mathrm{~A} 1650$ & 0.0843 & 1.56 & 379.24 \\
$\mathrm{~A} 1835$ & 0.2532 & 3.91 & 1267.62 \\
$\mathrm{~A} 2029$ & 0.0765 & 1.43 & 342.24 \\
Ophiuchus & 0.028 & 0.55 & 120.84 \\
\hline
\end{tabular}

Column 1: Cluster Name; Col. 2: Redshift; Col. 3: Angular to linear conversion; Col. 4: Luminosity distance.

on the basis of their extremely regular cluster-scale X-ray morphology and lack of evidence of a recent major merger. All have been well observed by the Chandra satellite. The X-ray data indicate that they all have cool, dense cores (see references in sections on individual clusters below). As often observed in these clusters (e.g., Markevitch et al. 2003), all exhibit signs of the sloshing of dense gas around their $\mathrm{cD}$ galaxy, the presumed center of the cluster gravitational potential. We also selected these cooling core clusters because of their intermediate redshifts $(z>0.05)$. This selection criterion ensures that a minihalo of $500 \mathrm{kpc}$ in extent would have an angular size smaller than $10^{\prime}$. Therefore, there would be no significant missing flux for the shortest of Very Large Array (VLA) baselines. We observed A1068, A1413, and A1650 at $1.4 \mathrm{GHz}$ with the VLA in C configuration, while A1835 and A2029 were observed both in $\mathrm{C}$ and $\mathrm{D}$ configurations. These data provided an excellent combination of resolution and sensitivity in studying the largescale cluster emission.

Calibration and imaging were performed with the NRAO Astronomical Image Processing System (AIPS) package. The data were calibrated in both phase and amplitude. The phase calibration was completed using nearby secondary calibrators, observed at intervals of $\sim 20 \mathrm{~min}$. The flux-density scale was calibrated by observing 3C 286. Brightness images were produced following the standard procedures: Fourier-Transform, Clean, and Restore. Self-calibration was applied to remove residual phase variations.

In $\mathrm{C}$ array, the $1 \sigma \mathrm{rms}$ noise reached in our observations range from $0.022 \mathrm{mJy} /$ beam (in A2029) to $0.045 \mathrm{mJy} / \mathrm{beam}$ (in A1650), while in D array the rms noise is of $0.025 \mathrm{mJy} / \mathrm{beam}$ in A1835 and $0.031 \mathrm{mJy} / \mathrm{beam}$ in A2029. The cluster A2029 also corresponds to the highest peak intensity and in this case we reached a extraordinary high dynamic range. The dynamic range, measured to be the ratio of the peak brightness to the rms noise, was 10850:1 and 14450:1 in the C and D configuration respectively.

To isolate the mini-halo emission from the radio emission of the central cD galaxy, we retrieved high resolution archival observations at $1.4 \mathrm{GHz}$ with the VLA in A configuration of A1825 and A2029 (program AT211 and AL252 respectively).

While a wide-field X-ray image of the Ophiuchus cluster from ROSAT PSPC suggests merging on a cluster-wide scale, the Chandra X-ray image indicates that its cool core is insignificantly disturbed (apart from the usual sloshing, Ascasibar \& Markevitch 2006), thus we decided to include this interesting cluster in our sample.

We retrieved an archival observation of the Ophiuchus cluster at $1.4 \mathrm{GHz}$ with the VLA in D configuration (program AC261). After precessing the original data to J2000 coordinates, the data were calibrated in phase and amplitude, and a cluster 
Table 2. Details of the VLA observations.

\begin{tabular}{|c|c|c|c|c|c|c|c|c|}
\hline Cluster & $\begin{array}{c}\text { RA } \\
\text { J2000 }\end{array}$ & $\begin{array}{c}\text { Dec } \\
\text { J2000 }\end{array}$ & $\begin{array}{c}\text { Frequency } \\
\mathrm{MHz}\end{array}$ & $\begin{array}{c}\text { Bandwidth } \\
\mathrm{MHz}\end{array}$ & Config. & $\begin{array}{l}\text { Obs. Time } \\
\mathrm{h}\end{array}$ & Date & Note \\
\hline A1068 & 104047.0 & +395718.0 & $1465 / 1415$ & 25 & $\mathrm{C}$ & $3.4,1.0,5.7$ & 2006 Oct. $21,23,28$ & \\
\hline A1413 & 115519.0 & +232430.0 & $1465 / 1415$ & 25 & $\mathrm{C}$ & 8.0 & 2006 Oct. 23 & \\
\hline A1650 & 125841.0 & -014525.0 & $1465 / 1415$ & 25 & $\mathrm{C}$ & 7.0 & 2006 Oct. 22 & \\
\hline A1835 & 140102.0 & +025130.0 & $1465 / 1385$ & 50 & D & 4.2 & 2003 March 10 & \\
\hline A1835 & " & " & $1465 / 1415$ & 25 & $\mathrm{C}$ & $0.7,4.7$ & 2006 Oct. 23,28 & \\
\hline A1835 & 140102.1 & +025242.7 & $1465 / 1665$ & 25 & A & 2 & 1998 Apr. 23 & archive (AT211) \\
\hline A2029 & 151058.0 & +054542.0 & $1465 / 1385$ & 50 & D & 4.7 & 2003 March 09 & \\
\hline A2029 & " & " & $1465 / 1415$ & 50 & $\mathrm{C}$ & 6.3 & 2006 Dec. 26 & \\
\hline A2029 & 151056.1 & 054442.6 & $1465 / 1515$ & 25 & A & 0.1 & 1993 Jan. 14 & archive (AL252) \\
\hline Ophiuchus & 171231.9 & -232032.6 & $1452 / 1502$ & 25 & D & 0.5 & 1990 Jan. 25 & archive (AC261) \\
\hline
\end{tabular}

Column 1: Cluster Name; Cols. 2, 3: observation pointing (RAJ2000, DecJ2000); Col. 4: observing frequency; Col. 5: bandwidth; Col. 6: VLA configuration; Col. 7: total integration time on source; Col. 8: observing dates; Col. 9: notes on archival observations.

radio image was obtained following standard procedures. The phase calibration was completed using the nearby secondary calibrator 1730-130, observed at intervals of $\sim 10 \mathrm{~min}$, while the flux-density scale was calibrated by observing $3 \mathrm{C} 286$. In this observation, we achieved a $1 \sigma \mathrm{rms}$ noise level of $0.1 \mathrm{mJy} / \mathrm{beam}$. At the distance of the Ophiuchus cluster, a mini-halo of $500 \mathrm{kpc}$ in extent would have an angular size of about $15^{\prime}$. This value is close to the maximum angular size observable at $1.4 \mathrm{GHz}$ by the VLA in D configuration.

\section{Results}

For each cluster, we report the results obtained in this campaign of observations. We find that in the central regions of A1835, A2029, and Ophiuchus, the dominant radio galaxy is surrounded by a diffuse low-brightness radio emission that we identify as a mini-halo. Low-significance indication of diffuse emission are present in A1068 and A1413, although their unambiguous classification as mini-halos would require further investigation. Finally, we detect no diffuse emission in A1650. We indicate the radio flux densities of both the mini-halos and the central point sources in A1835, A2029, and Ophiuchus, calculated by using the fit procedure described in Paper II, for more details of the density flux calculation, see that paper.

\subsection{Abell 1068}

A1068 is a relaxed cluster that has a peaked X-ray surface brightness profile, a declining temperature gradient, and an increasing gas metallicity toward the cluster center (Wise et al. 2004; McNamara et al. 2004), exhibiting many features commonly seen in other cooling-core clusters.

Figure 1 shows the central cluster radio emission compared with optical and X-ray images. In the top left panel, we present the optical cluster image with a radio contour plot overlaid. The total intensity radio contours are from the $1.4 \mathrm{GHz}$ image with a resolution of $15^{\prime \prime}$, while the optical image was taken from the POSS2 red plate in the Optical Digitalized Sky Survey ${ }^{2}$. The field of view of this image is about 3.5'. This panel suggests that diffuse emission on a scale larger than $100 \mathrm{kpc}$ may be present around the central radio emission. The central radio emission exhibits two peaks. The first coincides with the cluster $\mathrm{cD}$ galaxy, while the second is located toward the southwest at a distance of about $15^{\prime \prime}$ and is associated with another

\footnotetext{
${ }^{2}$ See http://archive.eso.org/dss/dss
}

bright cluster galaxy. The separation of these two radio galaxies is shown in the top right panel of Fig. 1, where the higher resolution $\left(\simeq 5^{\prime \prime}\right)$ FIRST image (Becker et al. 1995) is overlaid on the HST image. The radio emission associated with the $\mathrm{cD}$ galaxy has a flux density of $8.5 \pm 0.6 \mathrm{mJy}$, which corresponds to a power $L_{1.4 \mathrm{GHz}}=4.2 \times 10^{23} \mathrm{~W} / \mathrm{Hz}$. It is marginally resolved and extends to the north-west. The south-western source shows a head-tail morphology and has a flux density of $10.4 \pm 0.6 \mathrm{mJy}$.

To highlight the possible diffuse large-scale emission associated with the intra-cluster medium, we subtracted the flux density of the two central sources, calculated in the FIRST survey from our lower resolution image. The total radio emission estimated in the cluster central emission from our map was $22.3 \pm 0.7 \mathrm{mJy}$. Therefore, $3.4 \pm 1.1 \mathrm{mJy}$, which corresponds to a power $L_{1.4 \mathrm{GHz}}=1.7 \times 10^{23} \mathrm{~W} / \mathrm{Hz}$, appears to be associated with low diffusion emission. However, we note that the FIRST subtraction may be uncertain. A possible variation in the core-component density flux and/or any absolute calibration error between the FIRST and this dataset could cause in an under or over subtraction of flux, and therefore the residual flux associated with low diffusion emission must be interpreted with caution.

In the bottom panel, our radio image is overlaid on the Chandra X-ray emission of the cluster in the $0.5-4 \mathrm{keV}$ band. The X-ray emission appears elongated in the north-west southeast direction. The low surface brightness radio emission that is possibly associated with cluster diffuse emission appears to have the same orientation as both the cluster X-ray emission and the central cD galaxy.

In conclusion, a hint of diffuse emission has been detected in our data around the dominant cluster galaxy in A1068. However, given the uncertainty related to comparing datasets, this result requires further confirmation.

We note that in the field of view of this observation, we detected a giant radio galaxy that is not related to A1068, whose properties are shown in Appendix A.

\subsection{Abell 1413}

In the top left panel of Fig. 2, our deep radio observation at $1.4 \mathrm{GHz}$ with a resolution of $15^{\prime \prime}$ is overlaid on the POSS2 red plate image. The field of view of the image is about $3^{\prime}$. A diffuse low-surface-brightness emission of about $1.5^{\prime}(\simeq 220 \mathrm{kpc})$ in size was detected in close proximity to the cluster center. However, the radio-optical overlay clearly indicates that the peak of the 

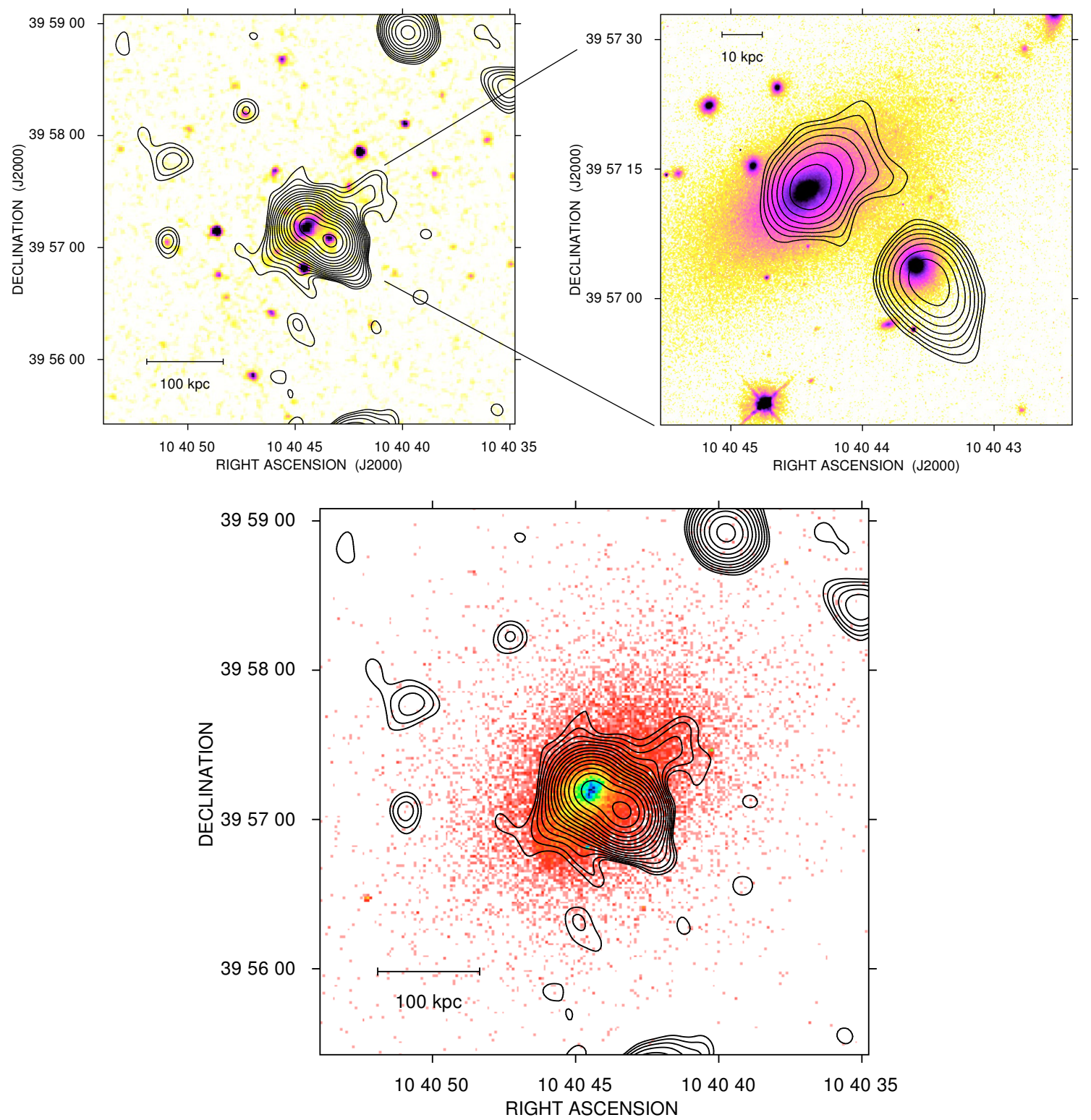

Fig. 1. Top, left: total intensity radio contours of A1068 at $1.4 \mathrm{GHz}$ with a $F W H M$ of $15^{\prime \prime} \times 15^{\prime \prime}$. The first contour level is drawn at $0.08 \mathrm{mJy} / \mathrm{beam}$ and the others are spaced by a factor $\sqrt{2}$. The sensitivity $(1 \sigma)$ is $0.027 \mathrm{mJy} / \mathrm{beam}$. The contours of the radio intensity are overlaid on the optical POSS2 image. Top, right: zoom of total intensity radio contours in the center of A1068 at $1.4 \mathrm{GHz}$ with a FWHM of 5.4" $\times 5.4^{\prime \prime}$ taken from the FIRST survey. The first contour level is drawn at $0.5 \mathrm{mJy} /$ beam and the rest are spaced by a factor $\sqrt{2}$. The sensitivity of the FIRST survey $(1 \sigma)$ is $0.15 \mathrm{mJy} / \mathrm{beam}$. The contours of the radio intensity are overlaid on the optical image taken from the HST (F606W filter) archive. Bottom: total intensity radio contours of A1068 at $1.4 \mathrm{GHz}$ with a $F W H M$ of $15^{\prime \prime} \times 15^{\prime \prime}$ overlaid on the Chandra X-ray image in the $0.5-4 \mathrm{keV}$ band.

central radio emission is offset to the east with respect to the central cD galaxy.

To investigate in detail the central radio emission of the cluster, in the top right panel of Fig. 2 we show a high resolution zoom of this region. In this panel, the image at $1.4 \mathrm{GHz}$ taken from the FIRST survey with a resolution of $\simeq 5^{\prime \prime}$ is overlaid on the HST image. The FIRST survey confirms that any discrete radio emission is associated with the central cD. We estimated a $3 \sigma$ flux limit to a point-source radio emission associated with the central cD galaxy of $0.45 \mathrm{mJy}$, which corresponds to a power $L_{1.4 \mathrm{GHz}}<2.4 \times 10^{22} \mathrm{~W} / \mathrm{Hz}$. A faint (flux density of $2.9 \pm 0.7 \mathrm{mJy}$ ), unresolved, radio source is detected that coincides with another galaxy to the east.
To estimate the flux density of diffuse emission on large scales, we subtracted the flux density of the faint radio source detected in the FIRST survey from that measured in our lower resolution image. The total radio emission estimated in the cluster central emission from our map was $4.8 \pm 0.2 \mathrm{mJy}$. Therefore $1.9 \pm 0.7 \mathrm{mJy}$, which corresponds to a power $L_{1.4 \mathrm{GHz}}=1.0 \times$ $10^{23} \mathrm{~W} / \mathrm{Hz}$, is indicative of low diffusion emission, but the low significance $(<3 \sigma)$ require further observations. As mentioned in the case of A1068, the FIRST flux density subtraction must also be considered with caution.

In the X-ray band, A1413 has been observed by XMM-Newton (Pratt \& Arnaud 2002) and Chandra (Baldi et al. 2007; Vikhlinin et al. 2005). It exhibits a regular morphology 

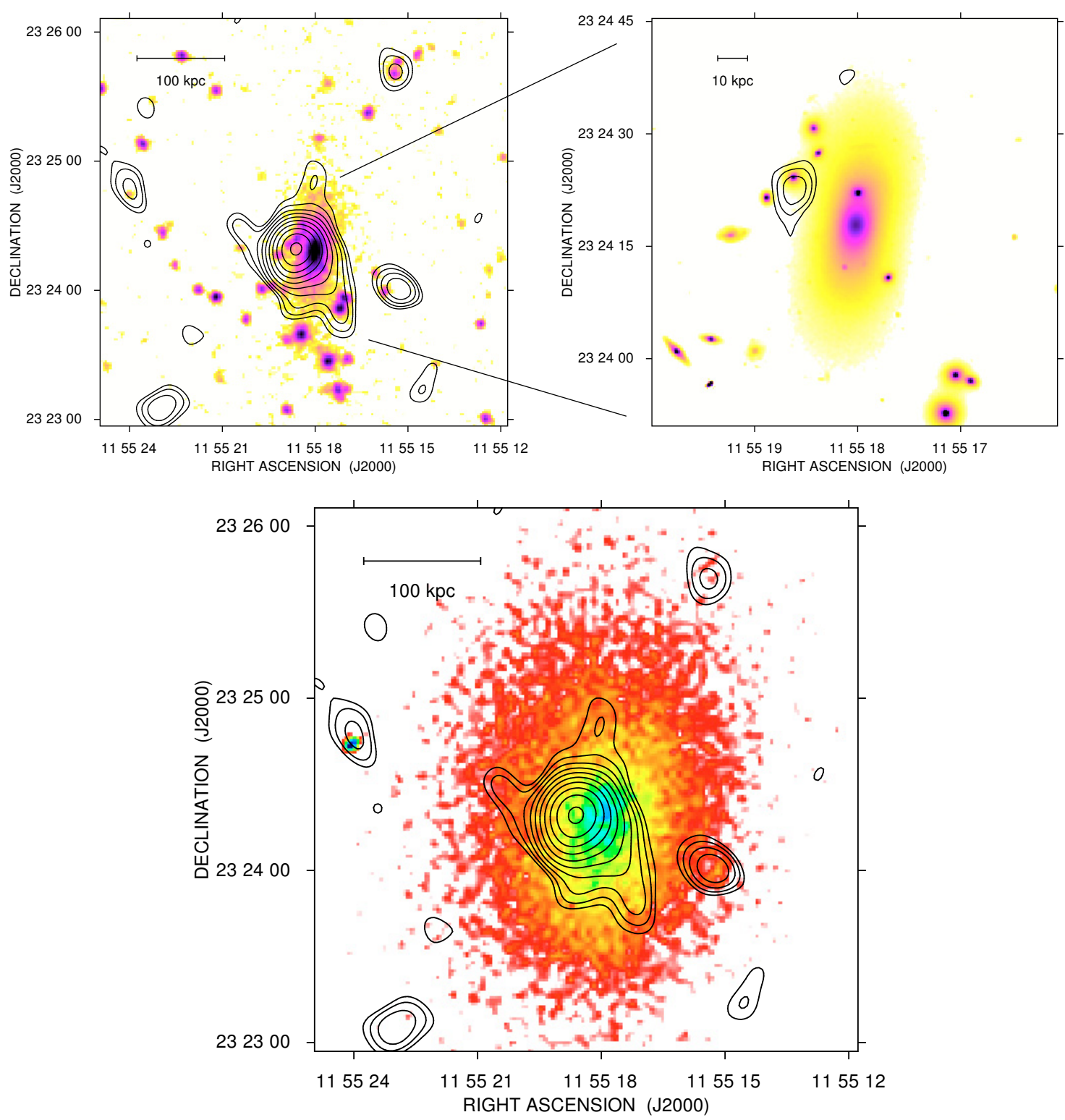

Fig. 2. Top, left: total intensity radio contours of A1413 at $1.4 \mathrm{GHz}$ with a $F W H M$ of $15^{\prime \prime} \times 15^{\prime \prime}$. The first contour level is drawn at $0.1 \mathrm{mJy} / \mathrm{beam}$ and the rest are spaced by a factor $\sqrt{2}$. The sensitivity $(1 \sigma)$ is $0.035 \mathrm{mJy} /$ beam. The contours of the radio intensity are overlaid on the optical POSS2 image. Top, right: zoom of total intensity radio contours in the center of A1413 at $1.4 \mathrm{GHz}$ with a FWHM of 5.4" $\times 5.4^{\prime \prime}$ taken from the FIRST survey. The first contour level is drawn at $0.5 \mathrm{mJy} /$ beam and the rest are spaced by a factor $\sqrt{2}$. The sensitivity of the FIRST survey $(1 \sigma)$ is $0.15 \mathrm{mJy} / \mathrm{beam}$. The contours of the radio intensity are overlaid on the optical image taken from the HST (F606W filter) archive. Bottom: total intensity radio contours of A1413 at $1.4 \mathrm{GHz}$ with a $F W H M$ of $15^{\prime \prime} \times 15^{\prime \prime}$ overlaid on the Chandra X-ray image in the $0.5-4 \mathrm{keV}$ band.

and no evidence of recent merging. In the bottom panel of Fig. 2, the previous radio image is overlaid on the Chandra X-ray emission of the cluster in the $0.5-4 \mathrm{keV}$ band. The orientation of the $\mathrm{X}$-ray emission is well aligned with that of the central cD galaxy, while the diffuse radio emission is offset from the X-ray peak and seems elongated in a slightly different direction. However this could be due to the confusion effect of the nearby unrelated discrete source visible in the FIRST image. As in the case of A1068, further observations are needed to confirm the presence of a mini-halo and the possible discrepancy between the radio and the $\mathrm{X}$-ray peak.

A peculiarity of the extended emission in A1413 is that the $\mathrm{cD}$ galaxy, located in the middle of this putative mini-halo, does not contain a compact radio source, at least at the FIRST sensitivity level. Another cluster with a similar characteristic is A2142 (Giovannini \& Feretti 2000). The properties of these clusters may suggest the presence of "relic" mini-halos in which the central cD galaxy is switched-off, while the diffuse mini-halo continues to emit. Observations at lower frequency might help in understanding this case.

\subsection{Abell 1650}

Studies of this cluster in X-ray (Takahashi \& Yamashita 2003; Donahue et al. 2005) pointed out that there is no clear evidence of substructures, suggesting that the cluster has not experienced a major merger in the recent past, and is in a relaxed state. However, while the central cooling time of this cluster is shorter 

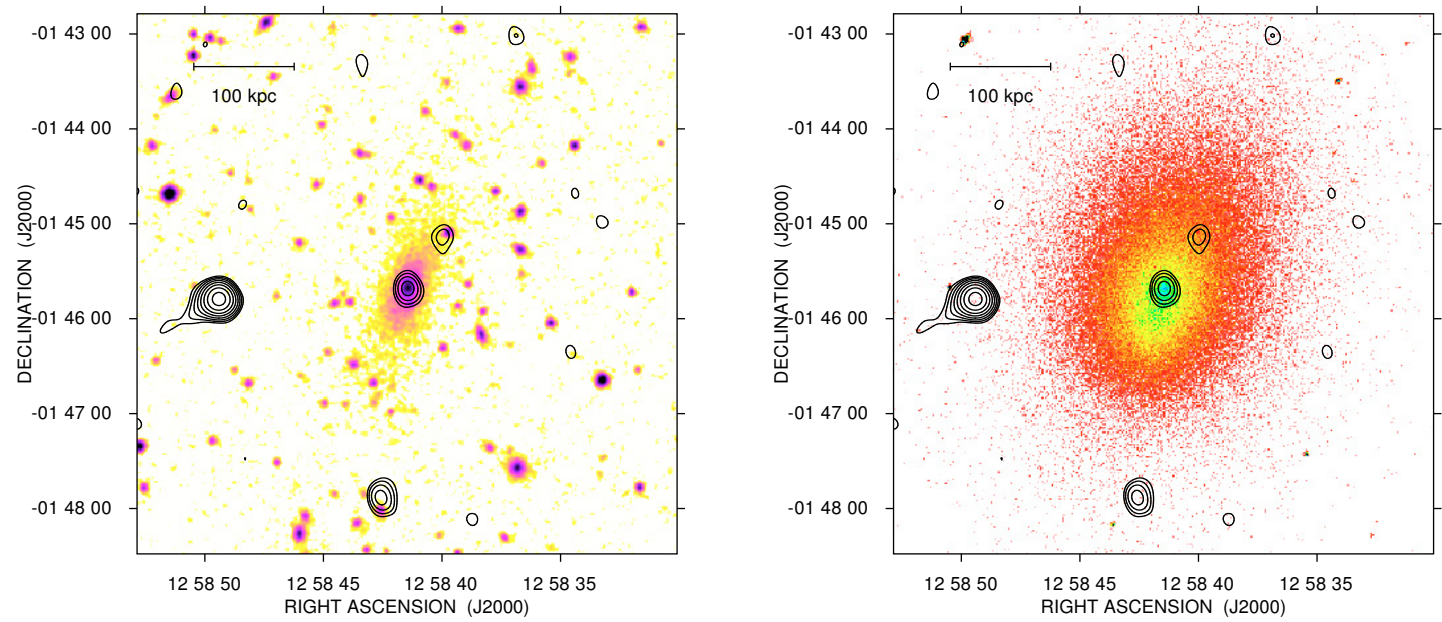

Fig. 3. Left: total intensity radio contours of A1650 at $1.4 \mathrm{GHz}$ with a $F W H M$ of $16^{\prime \prime} \times 16^{\prime \prime}$. The first contour level is drawn at $0.13 \mathrm{mJy} / \mathrm{beam}$ and the rest are spaced by a factor $\sqrt{2}$. The sensitivity $(1 \sigma)$ is $0.045 \mathrm{mJy} / \mathrm{beam}$. The contours of the radio intensity are overlaid on the optical POSS2 image. Right: total intensity radio contours of A1650 overlaid on the Chandra X-ray image in the $0.5-4 \mathrm{keV}$ band.

than the Hubble time and it has a strong metallicity gradient, it does not have a significant temperature gradient close to its center.

A1650 contains a single optically luminous central cD galaxy that is known to be radio quiet (Burns 1990). The FIRST survey does not detect any radio emission coincident with this dominant cluster galaxy.

We imaged this cluster at $1.4 \mathrm{GHz}$, reaching a sensitivity at $1 \sigma$ of $0.045 \mathrm{mJy} /$ beam. In Fig. 3, the central cluster radio emission is overlaid on both the POSS2 and the Chandra image. We note that our deep image reveals the presence of a faint, unresolved, radio source in coincidence with the $\mathrm{cD}$ galaxy. Its flux density is $0.44 \pm 0.09 \mathrm{mJy}$, which corresponds to a power $L_{1.4 \mathrm{GHz}}=7.6 \times 10^{21} \mathrm{~W} / \mathrm{Hz}$. However, at this sensitivity level, this cluster does not exhibit any signs of mini-halo. We estimated a $3 \sigma$ flux limit of $1.35 \mathrm{mJy}$ for a mini-halo extended $300 \mathrm{kpc}$ in size, which corresponds to a power of $L_{1.4 \mathrm{GHz}}<$ $2.3 \times 10^{22} \mathrm{~W} / \mathrm{Hz}$.

At the periphery of the A1650 cluster, we note that we detected an unusually round radio galaxy, whose properties are shown in Appendix B.

\subsection{Abell 1835}

The X-ray emission of A1835 has been studied with XMM (Peterson et al. 2001; Majerowicz et al. 2002) and Chandra (Schmidt et al. 2001; Markevitch 2002), and both sets of observations provided evidence that the cluster has a relatively cool (3-4 keV) inner core surrounded by a hotter $(8-9 \mathrm{keV})$ outer envelope. Overall, the X-ray image displays a relaxed morphology, although substructures have been detected at the cluster center.

In Fig. 4, we display the central cluster radio emission, obtained with the VLA in C configuration, superimposed on optical and X-ray images. In the top left panel, we present the optical cluster image with a radio contour plot overlaid. The total intensity radio contours are at $1.4 \mathrm{GHz}$ with a resolution of $16^{\prime \prime}$, while the optical image was extracted from the POSS2 red plate in the Optical Digitalized Sky Survey. The field of view of this image is about $4^{\prime}$. Our image clearly displays evidence of extended emission around the central radio core.
The peak of the central radio emission is coincident with the cluster $\mathrm{cD}$ galaxy. The emission as detected at higher resolution is shown in the top right panel of Fig. 4, in which we overlay the high resolution radio image from the VLA archive (project AT211) on the HST image. The sensitivity of the image is $0.037 \mathrm{mJy} /$ beam and the resolution is $1.36^{\prime \prime} \times 1.30^{\prime \prime}$.

In the bottom left panel, our $16^{\prime \prime}$ resolution radio image is overlaid on the Chandra X-ray emission of the cluster in the 0.5-4 keV band. The diffuse radio emission appears extended to several hundreds of kiloparsecs and appears well mixed with the thermal plasma. The round morphology of the diffuse emission also coincides well with the morphology of the thermal gas.

The bottom right panel displays a large field of view of the total intensity radio contours of A1835 at $1.4 \mathrm{GHz}$ with a $F W H M$ of $53^{\prime \prime} \times 53^{\prime \prime}$. This image was obtained with the VLA in D configuration. Despite the presence of several radio galaxies located close to the cluster center, a new mini-halo in A1835 is clearly evident at this lower resolution.

The mini-halo flux density is $6.0 \pm 0.8 \mathrm{mJy}$, which corresponds to a power $L_{1.4 \mathrm{GHz}}=1.2 \times 10^{24} \mathrm{~W} / \mathrm{Hz}$. The central radio source has a flux density of $32.2 \pm 0.8$, which corresponds to a power $L_{1.4 \mathrm{GHz}}=6.2 \times 10^{24} \mathrm{~W} / \mathrm{Hz}$, which agrees with Bîrzan et al. (2008).

\subsection{Abell 2029}

The nearby well-studied cluster of galaxies A2029, is one of the most optically detected regular rich clusters known and is dominated by a central ultra-luminous cD galaxy (Dressler 1978; Uson et al. 1991).

The hot cluster A2029 has been extensively studied in X-rays (e.g., Buote \& Canizares 1996; Sarazin et al. 1998; Lewis et al. 2002; Clarke et al. 2004; Vikhlinin et al. 2005; Bourdin \& Mazzotta 2008). On scales $r>100-200 \mathrm{kpc}$, it is one of the most regular and relaxed clusters known (e.g., Buote \& Tsai 1996). However, in the image of the cool dense core, Clarke et al. (2004) observed a subtle spiral structure. Along with several sharp brightness edges, it indicates ongoing gas sloshing that is most likely indicative of past subcluster infall episodes (Ascasibar \& Markevitch 2006). As in many cool-core clusters with central radio sources, some small-scale X-ray structure is 

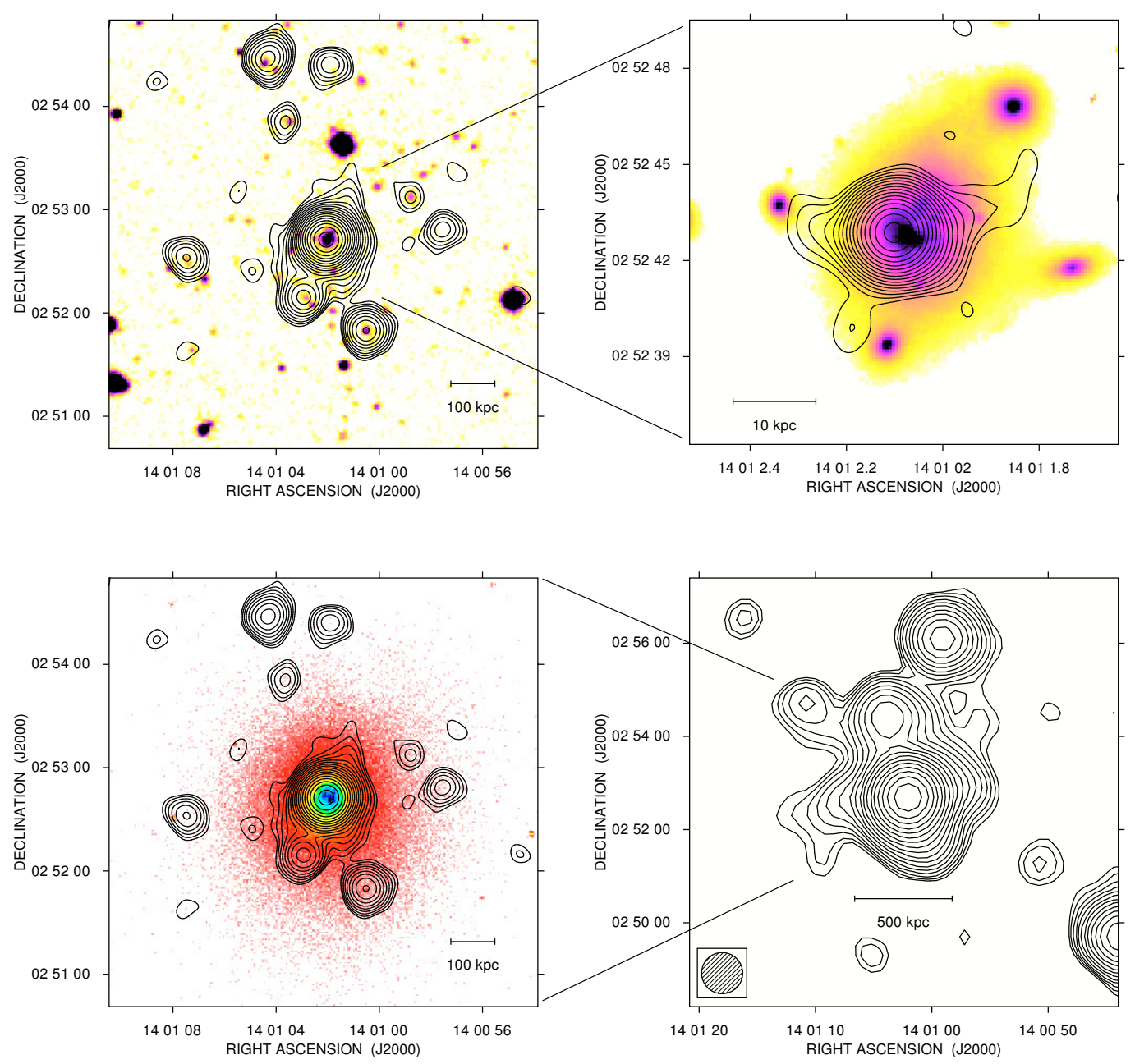

Fig. 4. Left: total intensity radio contours of A1835 at $1.4 \mathrm{GHz}$ with a FWHM of $16^{\prime \prime} \times 16^{\prime \prime}$. The first contour level is drawn at $0.1 \mathrm{mJy} / \mathrm{beam}$ and the rest are spaced by a factor $\sqrt{2}$. The sensitivity $(1 \sigma)$ is $0.036 \mathrm{mJy} / \mathrm{beam}$. The contours of the radio intensity are overlaid on the optical POSS2 image (top) and on the Chandra X-ray image in the $0.5-4 \mathrm{keV}$ band (bottom). Right, top: zoom of total intensity radio contours in the center of A1835 at $1465 \mathrm{MHz}$ with a FWHM of $1.36^{\prime \prime} \times 1.30^{\prime \prime}\left(\mathrm{PA}=2.5^{\circ}\right.$ ) taken from a VLA archival data set (AT211). The first contour level is drawn at $0.12 \mathrm{mJy} / \mathrm{beam}$ and the rest increase by a factor $\sqrt{2}$. The sensitivity of the high resolution image $(1 \sigma)$ is $0.037 \mathrm{mJy} / \mathrm{beam}$. The contours of the radio intensity are overlaid on the optical image taken from the HST ( $F 702 \mathrm{~W}$ filter) archive. Right, bottom: larger field of view of the total intensity radio contours of A1835 at $1.4 \mathrm{GHz}$ with a $F W H M$ of $53^{\prime \prime} \times 53^{\prime \prime}$. The first contour level is drawn at $0.1 \mathrm{mJy} /$ beam and the rest are spaced by a factor $\sqrt{2}$. The sensitivity $(1 \sigma)$ is $0.025 \mathrm{mJy} /$ beam.

also apparently connected with the central radio galaxy (Clarke et al. 2004).

In the top left panel of Fig. 5, our deep radio observation at $1.4 \mathrm{GHz}$ with a resolution of $16^{\prime \prime}$, obtained with the VLA in $\mathrm{C}$ configuration, is overlaid on the POSS2 red plate image. The field of view of the image is about $7^{\prime}$. A large-scale diffuse emission, most likely a mini-halo, is located around, but has its centroid offset from the central PKS1509+59 source, which is coincident with the cluster $\mathrm{cD}$ galaxy. The mini-halo is far more extended than the central radio galaxy and appears to have a filamentary morphology slightly elongated in a north-east to southwest direction. The relatively high resolution of our new image ensures that the detected diffuse emission is real and not due to a blend of discrete sources. A sign of a diffuse, extended emission in this cluster is also found by Marković et al. (2003) in a VLA observation at $74 \mathrm{MHz}$.

To distinguish more effectively between the mini-halo emission and the radio emission of the central $\mathrm{cD}$ galaxy, in the top right panel of Fig. 5, we show a high resolution zoom of the cluster central region. In this panel, the image at $1.4 \mathrm{GHz}$ with a resolution of $1.62^{\prime \prime} \times 1.35^{\prime \prime}$ taken from an archive data set (AL252) is overlaid on the HST image. As shown in this panel, the radio source PKS1509+59 associated with the central cD galaxy has a distorted morphology and two oppositely directed jets. The source has also a very steep integrated spectrum and a high rotation measure as discussed in detail by Taylor et al. (1994).

In the bottom left panel of Fig. 5, the previous radio image is overlaid on the Chandra X-ray emission of the cluster in the $0.5-4 \mathrm{keV}$ band. We retrieved archival X-ray data for comparison of the gas distribution and the cluster radio emission. The mini-halo appears elongated in the same direction as both the cluster X-ray emission and the central cD galaxy.

The bottom right panel shows the total intensity radio contours of A2029 at $1.4 \mathrm{GHz}$ for a larger field of view with a $F W H M$ of $53^{\prime \prime} \times 53^{\prime \prime}$. This image was obtained with the VLA in D configuration. The mini-halo in A2029 is even clearer in this lower resolution image. 

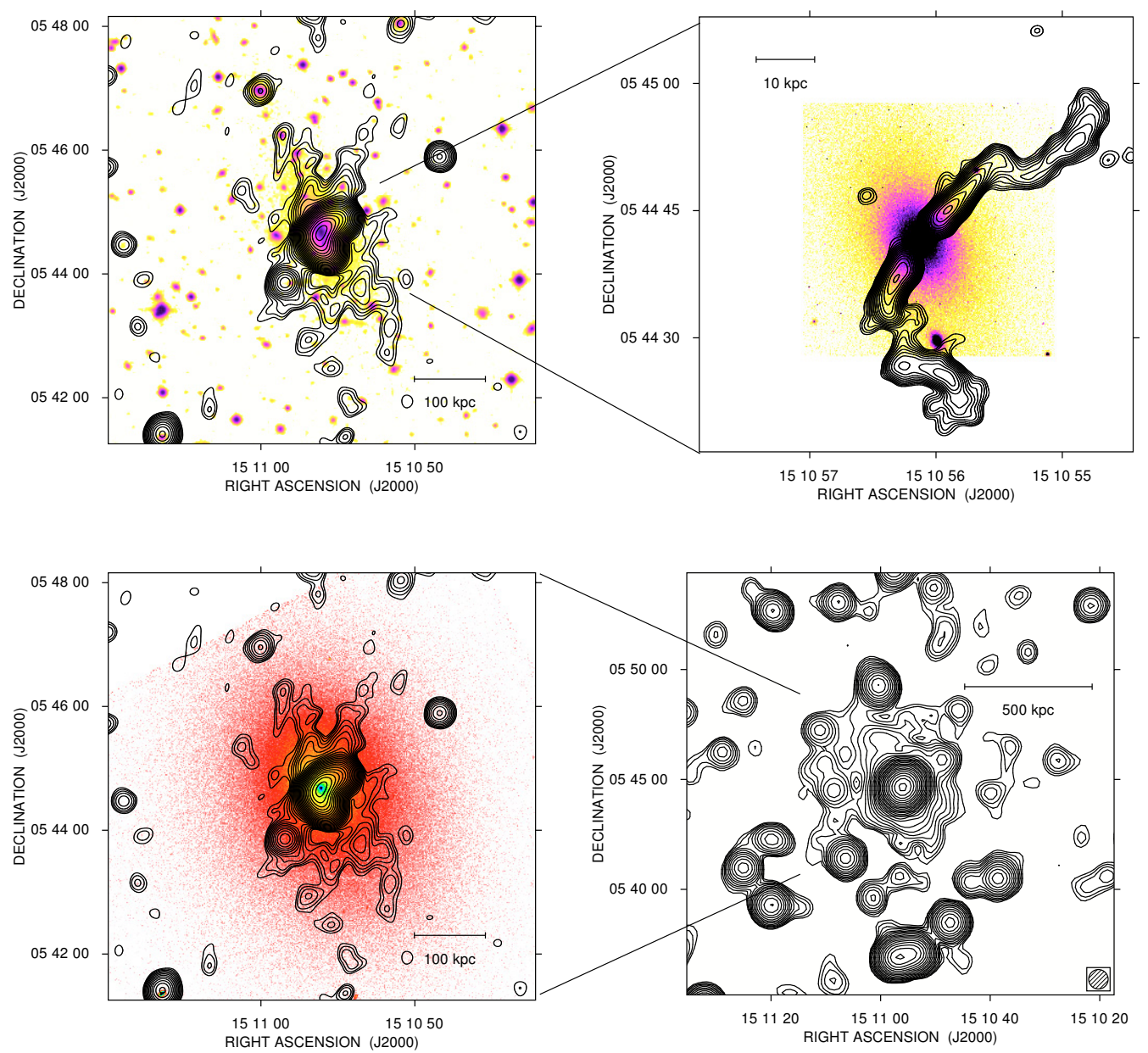

Fig. 5. Left: total intensity radio contours of A2029 at $1.4 \mathrm{GHz}$ with a $F W H M$ of $16^{\prime \prime} \times 16^{\prime \prime}$. The first contour level is drawn at $0.07 \mathrm{mJy} / \mathrm{beam}$ and the rest are spaced by a factor $\sqrt{2}$. The sensitivity $(1 \sigma)$ is $0.022 \mathrm{mJy} /$ beam. The contours of the radio intensity are overlaid on the optical POSS2 image (top) and on the Chandra X-ray image in the $0.5-4 \mathrm{keV}$ band (bottom). Right, top: zoom of the total intensity radio contours in the center of A2029 at $1.4 \mathrm{GHz}$ with a $F W H M$ of $1.62^{\prime \prime} \times 1.35^{\prime \prime}\left(\mathrm{PA}=-48.8^{\circ}\right)$ taken from a VLA archive data set (AL252). The first contour level is drawn at $0.25 \mathrm{mJy} / \mathrm{beam}$ and the rest are spaced by a factor $\sqrt{2}$. The sensitivity of the high resolution image $(1 \sigma)$ is $0.083 \mathrm{mJy} / \mathrm{beam}$. The contours of the radio intensity are overlaid on the optical image taken from the HST ( $F 450 \mathrm{~W}$ filter) archive. Right, bottom: larger field of view of the total intensity radio contours of A2029 at $1.4 \mathrm{GHz}$ with a $F W H M$ of $53^{\prime \prime} \times 53^{\prime \prime}$. The first contour level is drawn at $0.1 \mathrm{mJy} /$ beam and the rest are spaced by a factor $\sqrt{2}$. The sensitivity $(1 \sigma)$ is $0.031 \mathrm{mJy} /$ beam.

The mini-halo flux density is $18.8 \pm 1.3 \mathrm{mJy}$, which corresponds to a power $L_{1.4 \mathrm{GHz}}=2.6 \times 10^{23} \mathrm{~W} / \mathrm{Hz}$. While the central radio source has a flux density of $480.0 \pm 19.5 \mathrm{mJy}$, which corresponds to a power $L_{1.4 \mathrm{GHz}}=6.7 \times 10^{24} \mathrm{~W} / \mathrm{Hz}$.

\subsection{Ophiuchus}

Ophiuchus is a nearby, rich cluster located $12 \mathrm{deg}$ from the Galactic center. In X-rays, it is one of the hottest clusters known.

Suzaku data (Fujita et al. 2008) and the archival Chandra data indicate that this hot cluster has a cool, dense core. The archival wide-field ROSAT PSPC image suggests an ongoing unequal-mass merger, but the Chandra image indicates that the core is still largely undisturbed, although it exhibits the prototypical cold fronts caused by gas sloshing (Ascasibar \& Markevitch 2006). Eckert et al. (2008) detected, with INTEGRAL, Xray emission at high energies in excess of a thermal plasma spectrum with the cluster's mean temperature. This emission may be of non-thermal origin, caused by for example, by Compton scattering of relativistic electrons by the cosmic microwave background radiation (see e.g., Rephaeli et al. 2008;
Petrosian et al. 2008, and references therein for reviews). It would be particularly interesting to investigate the presence of relativistic electrons in the intergalactic medium of this cluster because by assuming that the synchrotron emission and the hard $\mathrm{X}$-ray excess are cospatial and produced by the same population of relativistic electrons, their detection would allow the determination of the cluster magnetic field.

We analyzed a VLA archive observation at $1.4 \mathrm{GHz}$ in D configuration. In the left panel of Fig. 6, the radio observation at $1.4 \mathrm{GHz}$ with a resolution of $91.4^{\prime \prime} \times 40.4^{\prime \prime}$ is overlaid on the POSS2 red plate image. The asymmetric beam is due to the far southern declination of the cluster. The field of view of the image is about $20^{\prime}$. It is evident that a large-scale low surface brightness diffuse emission, which is probably a mini-halo, is located around the central cD galaxy. We note that we may have missed some extended flux due to the large angular extension of the mini-halo and/or because of the poor uv coverage in the short VLA observation (only $0.5 \mathrm{~h}$ ).

The mini-halo flux density, calculated on the basis of the fit analysis described in Paper II, is $106.4 \pm 10.4 \mathrm{mJy}$, which corresponds to a power $L_{1.4 \mathrm{GHz}}=1.9 \times 10^{23} \mathrm{~W} / \mathrm{Hz}$. The same 

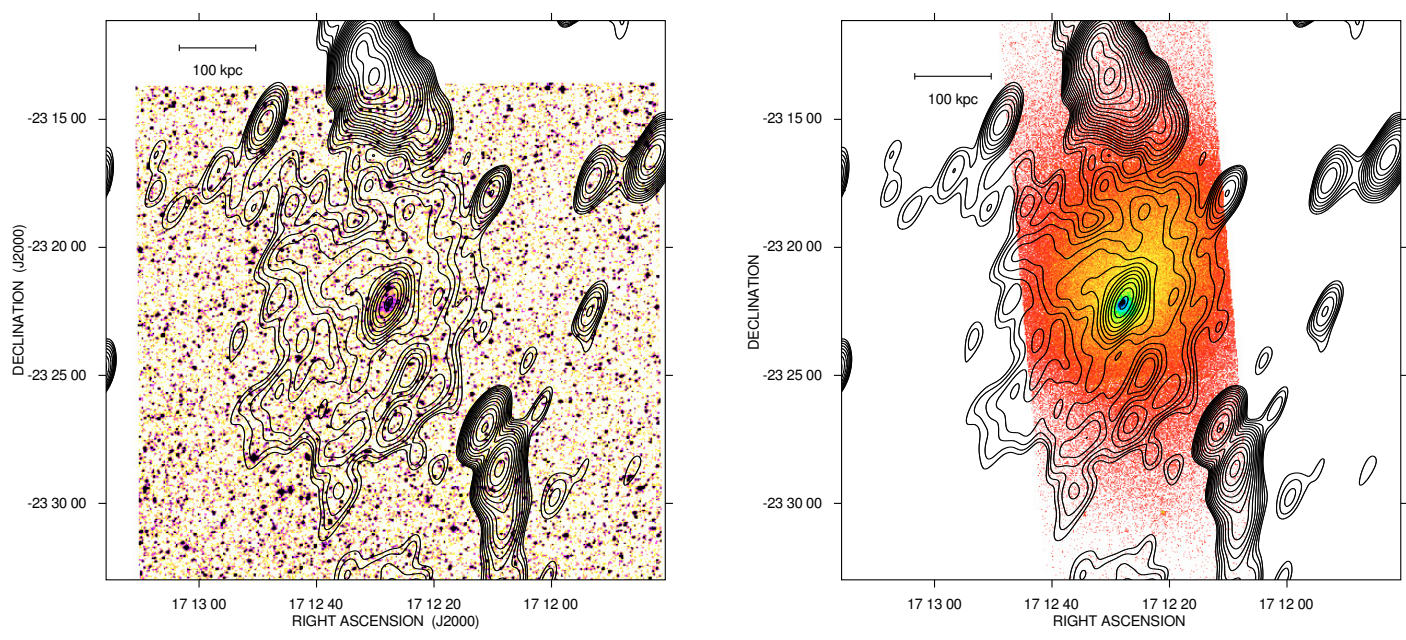

Fig. 6. Left: total intensity radio contours of Ophiuchus at $1.4 \mathrm{GHz}$ with a $F W H M$ of $91.4^{\prime \prime} \times 40.4^{\prime \prime}, \mathrm{PA}=-24.4^{\circ}$. The first contour level is drawn at $0.3 \mathrm{mJy} / \mathrm{beam}$ and the rest are spaced by a factor $\sqrt{2}$. The sensitivity $(1 \sigma)$ is $0.1 \mathrm{mJy} / \mathrm{beam}$. The contours of the radio intensity are overlaid on the optical POSS2 image. Right: total intensity radio contours of Ophiuchus overlaid on the Chandra X-ray image in the $0.5-4 \mathrm{keV}$ band.

analysis was used to estimate the radio flux density of the central $\mathrm{cD}$ galaxy, since no high resolution data for this cluster is available in the VLA archive. We obtained a flux density of $29 \pm$ $2 \mathrm{mJy}$, which corresponds to a power $L_{1.4 \mathrm{GHz}}=5.1 \times 10^{22} \mathrm{~W} / \mathrm{Hz}$.

In the right panel of Fig. 6, our radio image is overlaid on the Chandra X-ray emission of the cluster in the $0.5-4 \mathrm{keV}$ band. The peak of the X-ray emission is coincident with the position of the $\mathrm{cD}$ galaxy. Although the Chandra detector does not cover the entire cluster X-ray extension, a connection between the minihalo and the X-ray emission is evident, which is better investigated in the next section.

\section{Comparison between mini-halos and the X-ray emitting gas}

By superimposing the radio and X-ray data presented in previous sections, we emphasized the similarity between the radio minihalo emission and the cluster X-ray morphologies of A2029, A1835, and Ophiuchus. Because of the large angular extension of Ophiuchus, it is possible to perform a quantitative comparison between the radio and X-ray brightness. Here we compare the Chandra count rate image in the $0.5-4 \mathrm{keV}$ band, with the VLA radio image, corrected for the primary beam attenuation.

We first constructed a square grid covering a region containing both the radio mini-halo and the X-ray emission from which we excluded areas containing discrete radio sources. The grid cells size was chosen to be as large as the radio beam $\left(\simeq 90^{\prime \prime}\right)$. In the statistical analysis, all the discrete sources were excluded by masking them out. All pixels lying in blanked areas or close to the edges of the chips were also excluded from the statistics. A scatter plot of the radio versus X-ray brightness comparisons is presented in top panel of Fig. 7. Each point represents the mean brightness in each cell of the grid, while the error bars indicate the corresponding statistical error. The close similarity between radio and X-ray structures in Ophiuchus is demonstrated by the correlation between these two parameters. We fitted the data with a power law relation of the type: $I_{1.4 \mathrm{GHz}} \propto I_{X}^{1.51 \pm 0.04}$. We then present the same correlation this time obtained by azimuthally averaging the radio and the X-ray emission in annuli (see middle panel of Fig. 7). The data are fitted by a power law relation of the type $I_{1.4 \mathrm{GHz}} \propto I_{X}^{1.6 \pm 0.1}$, which is consistent within the errors with the scatter plot analysis. The correlation indicates that the radial decline in the non-thermal radio component is slightly steeper than that of the thermal X-ray component, as can be seen in the bottom panel of Fig. 7.

This trend may be explained by secondary models of minihalos, where a super-linear power law relation is expected (Dolag \& Enßlin 2000; Pfrommer et al. 2008). However, this result must be viewed with uncertainty, because as previously mentioned, we may miss part of the radio emission on the largest angular scales, which would reduce the slope of that relation. Moreover, especially in cooling-core clusters, the slope of the radius versus the $\mathrm{X}$-ray brightness may be affected by strongly variable temperature across the cluster.

It is interesting to compare the energetics of the mini-halos with that of the thermal X-ray emitting gas. It has become clear that AGNs play a crucial role in reheating the gas in the cores of clusters (e.g., McNamara \& Nulsen 2007). This decelerates the cooling and condensing of thermal gas onto the $\mathrm{cD}$ galaxy at the center of the cluster. The details of reheating are still misunderstood, since the volume occupied by the radio jets and lobes is small compared to that of the cool core. The mini-halo, however, appears well-matched to the cluster core in extent, even corresponding in elongation and surface brightness in some cases (e.g., Ophiucus, A2029).

Applying standard minimum energy arguments to the radio emission from the mini-halos and assuming that $k / f=1$ (where $k$ is the ratio of the relativistic particle energy to that in electrons emitting synchrotron radiation, and $f$ is the volume filling factor of magnetic field and relativistic particles) we infer a typical equipartition magnetic field strength of $0.6 \mu \mathrm{G}$ (but see Paper II for more detailed calculations), and a total energy of roughly $6 \times 10^{57} \mathrm{erg}$ for a typical mini-halo of radius $150 \mathrm{kpc}$. If we adopt a timescale for resupply of $10^{7}$ years then we derive an energy dissipation of $2 \times 10^{43} \mathrm{erg} / \mathrm{s}$. This is smaller by a factor 50 than the $10^{45} \mathrm{erg} / \mathrm{s}$ required to balance cooling in the X-rays (Fabian 1994). A possible solution is to increase the value of $k / f$. Dunn et al. (2005) measured a wide range of $k / f$ values in clusters with values for the mini-halos typically $\sim 100$, though they did not single out that class of objects. Since the total energy increases only by $(k / f)^{4 / 7}$, increasing $k / f$ by a factor of 100 only increases the total energy by a factor $\sim 14$, leaving a 

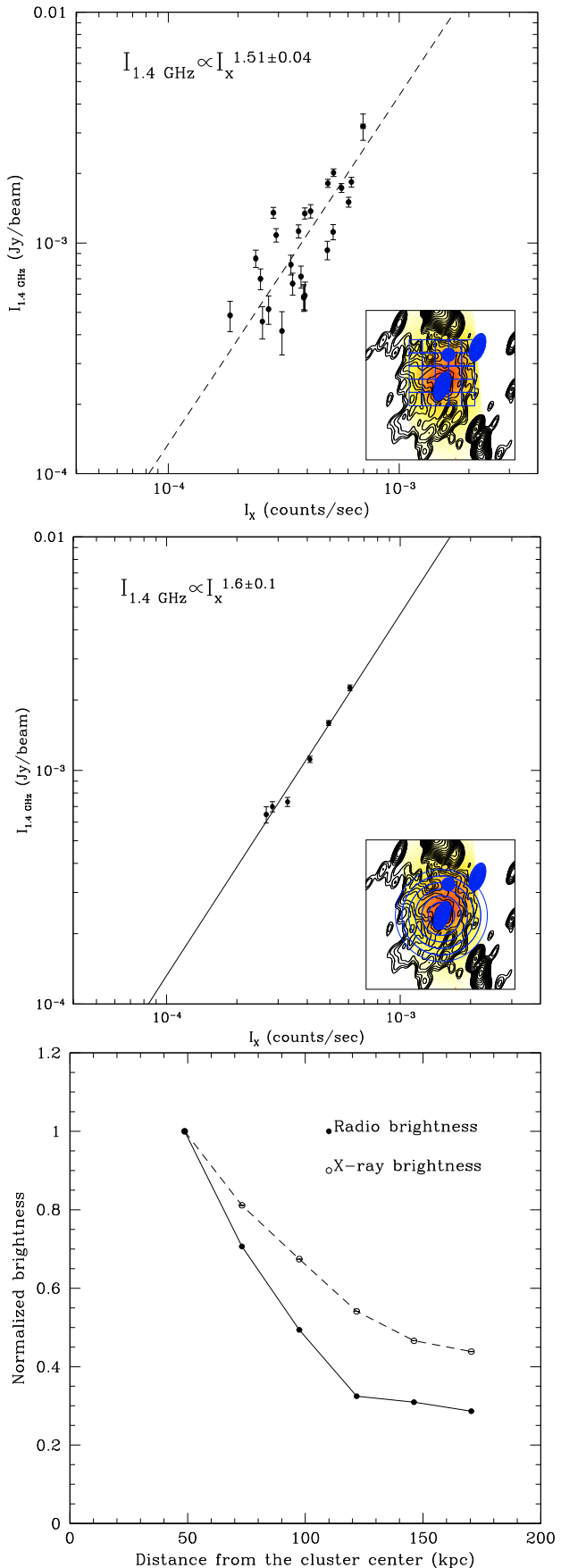

Fig. 7. Comparison of the radio and X-ray brightness in the Ophiuchus galaxy cluster. The scattered plot of the radio intensity at $1.4 \mathrm{GHz}$ versus the Chandra X-ray count rate is shown in the top panel. Each point represents the average in a rectangular region of $90^{\prime \prime} \times 90^{\prime \prime}$ as shown in the inset. The middle panel presents the same correlation this time obtained by azimuthally averaging the radio and the X-ray emission in annuli as shown in the inset. The normalized X-ray and radio radial profiles are shown in the bottom panel. All pixels lying in blanked areas or close to the edges of the chips have been excluded from the statistics.

factor $\sim 3.5$ unexplained. Given the various approximations employed, this is fairly close to the energy required. However, it raises the questions of (1) what process supplies energy to the mini-halos?; and (2) how do the mini-halos couple with the thermal gas? Even if the mini-halos are not involved in the heating of the cluster, they could be tracing where the heating is going on. One possibility is that the mechanism that heats the

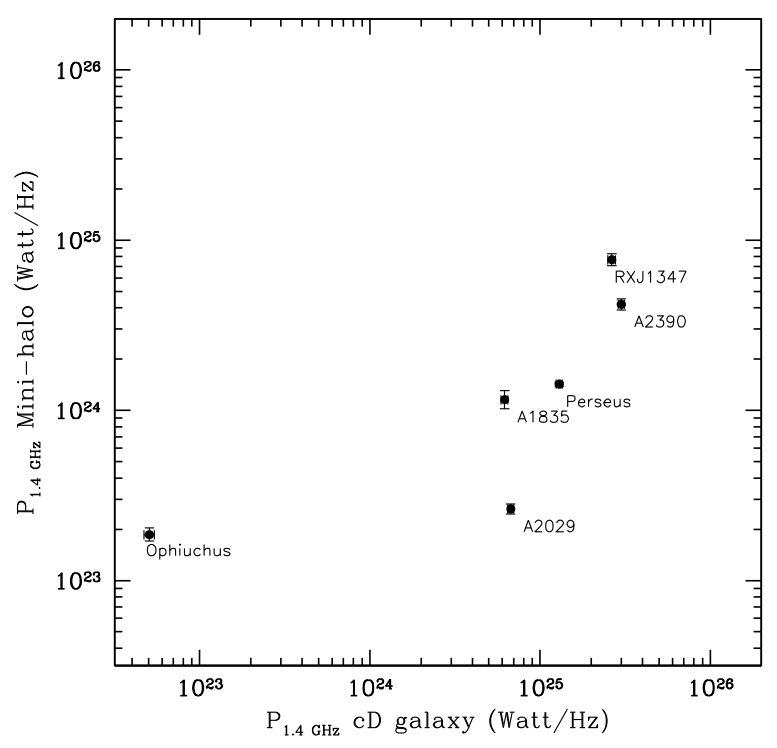

Fig. 8. Comparison between the radio power at $1.4 \mathrm{GHz}$ of the minihalos with that of the central $\mathrm{cD}$ galaxies, for the clusters analyzed in this work. All the fluxes are derived from the fit procedure described in Paper II.

cluster could also deposit some energy into relativistic electrons that then radiate in the radio.

\section{Comparison between the mini-halos and the central $C D$ radio galaxies}

As shown by Burns (1990), a large number of cooling core clusters contain powerful radio sources associated with central $\mathrm{cD}$ galaxies. Best et al. (2007) showed that brightest group and cluster galaxies are more likely to host a radio-loud AGN than other galaxies of the same stellar mass.

Since mini-halos are located at the center of cooling core clusters, where a radio-loud AGN is in general present, a possible link between the radio emission of the cDs and the minihalos is interesting to investigate in the framework of the models attempting to explain the formation of mini-halos.

In Fig. 8, we plot the radio power at $1.4 \mathrm{GHz}$ of the mini-halos versus those of the central $\mathrm{cD}$ galaxies. In addition to data for A1835, A2029 and Ophiuchus we plot data for RXJ1347.5-1145 (Gitti et al. 2007), A2390 (Bacchi et al. 2003), and Perseus (Pedlar et al. 1990). All fluxes are calculated in a consistent way from the fit procedure presented in Paper II.

The comparison between the radio power of mini halos and that of the central cD galaxy, indicates that there is a weak tendency for more powerful mini-halos to host stronger central radio sources. We recall that in a few clusters with cooling flows, the $\mathrm{cD}$ galaxy can be a low power radio source or even radio quiet. This is indicative of a recurrent radio activity with a duty cycle of AGN activity that is lower than the cooling time and lower than the radio mini-halo time life. Therefore, we do not expect a strong connection between $\mathrm{cD}$ and mini-halo radio power, and the position of Ophiuchus in the diagram implies that this cluster is undergoing a low radio activity phase of the central cD. We propose that this is important and that further studies and more robust statistical analyses are necessary to prove that minihalo emission is directly triggered by the central cD galaxy. 


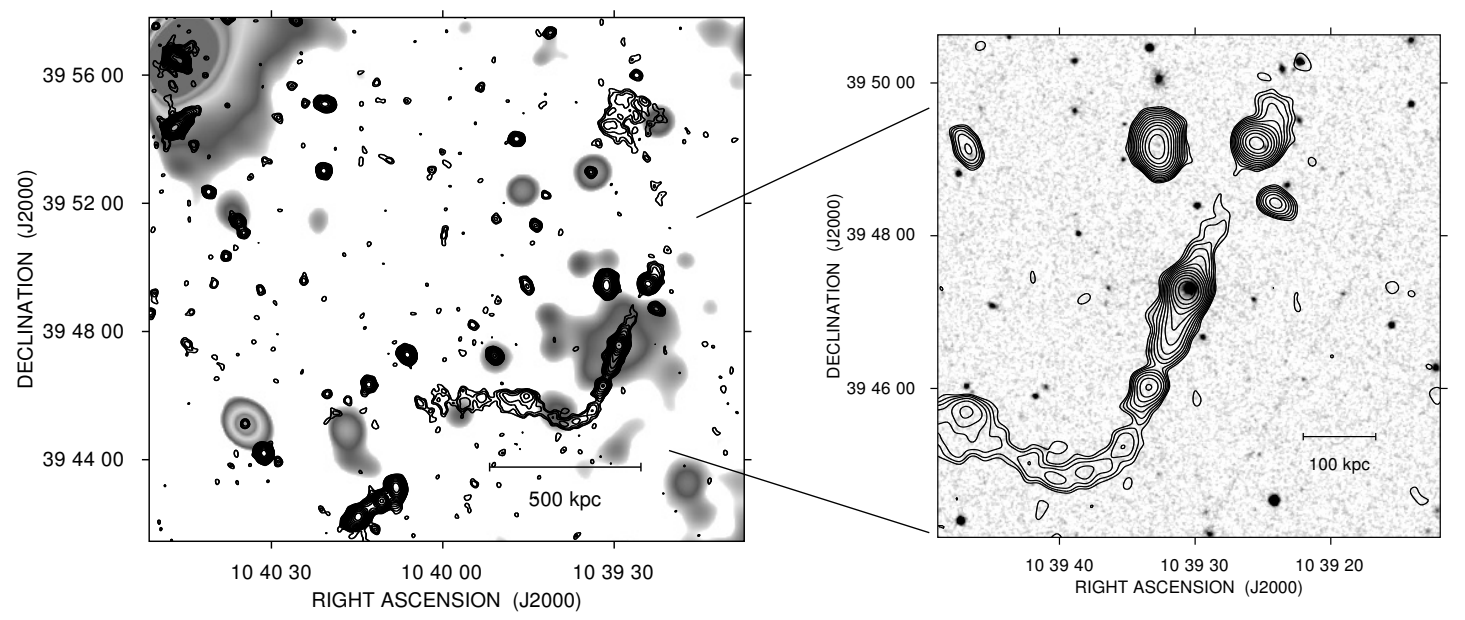

Fig. A.1. Total intensity radio contours on a large field of view on south-west of the A1068 cluster, at $1.4 \mathrm{GHz}$ with a FWHM of $15^{\prime \prime} \times 15^{\prime \prime}$ The first contour level is drawn at $0.065 \mathrm{mJy} / \mathrm{beam}$ and the rest are spaced by a factor $\sqrt{2}$. The sensitivity near the location of the giant radio galaxy $(1 \sigma)$ is $0.022 \mathrm{mJy} / \mathrm{beam}$. On the left the radio contours are overlaid on the XMM image in the 0.2-5.0 keV, convolved with a Gaussian $\sigma=20^{\prime \prime}$. On the right a zoom of the radio contours are overlaid on the optical POSS2 image.

\section{Conclusions}

Mini-halos in clusters are still poorly understood sources. They are a rare phenomenon, which have been found so far in only a few clusters. A larger number of mini-halo discoveries and more information about their physical properties is necessary to discriminate between the different mechanisms suggested for transferring energy to the relativistic electrons that power the radio emission.

To search for new mini-halos, we have analyzed deep radio observations of A1068, A1413, A1650, A1835, A2029, and Ophiuchus, carried out with the Very Large Array at $1.4 \mathrm{GHz}$.

We have found that at the center of the clusters A1835, A2029, and Ophiuchus, the dominant radio galaxy is surrounded by a diffuse low surface brightness mini-halo. The relatively high resolution of our new images ensures that the detected diffuse emission is real and not due to a blend of discrete sources.

We analyzed the interplay between the mini-halos and the cluster X-ray emission. We identified a similarity between the shape of the radio mini-halo emission and the cluster X-ray morphology of A2029, A1835, and Ophiuchus. We note that, although all these clusters are considered to be relaxed systems, when analyzed in detail they are found to contain peculiar X-ray features at the cluster center, which are indicative of a link between the mini-halo emission and some minor merger activity. Because of the large angular extension of Ophiuchus, it is possible to perform a point-to-point comparison of the radio and $\mathrm{X}$-ray brightness distributions. The close similarity between radio and X-ray structures in this cluster is demonstrated by the correlation between these two parameters. We fitted the data with a power law relation of the type $I_{1.4 \mathrm{GHz}} \propto I_{X}^{1.51 \pm 0.04}$.

A hint of diffuse emission at the center of A1068 and A1413 is present with low significance $(2-3 \sigma)$, and before classification as mini-halos, further investigation is needed. In addition, in the field of view of one of our observations, we report the serendipitous detection of a giant radio galaxy, located at $\mathrm{RA}=10^{\mathrm{h}} 39^{\mathrm{m}} 30^{\mathrm{s}}$ $\operatorname{Dec}=39^{\circ} 47^{\prime} 19^{\prime \prime}$, of a total extension $\sim 1.6 \mathrm{Mpc}$ in size.

Finally, the comparison between the radio power of mini halos and that of the central cD galaxy, reveals that there is a weak tendency for the more powerful mini-halos to host stronger central radio sources.
Discriminating between the different scenarios proposed for mini-halo formation is difficult using present radio data. We note that the radio- $X$ ray connection in the Ophiuchus cluster may support secondary models. On the other hand, the analyses of the clusters studied here appear to indicate that mini-halos are not a common phenomenon in relaxed system, a result that is in closer agreement with primary models.

Acknowledgements. F.G. and M.M. thank the hospitality of the Harvard-Smithsonian Center for Astrophysics where most of this work was done. Support was provided by Chandra grants GO5-6123X and GO67126X, NASA contract NAS8-39073, and the Smithsonian Institution. This research was partially supported by ASI-INAF I/088/06/0 - High Energy Astrophysics and PRIN-INAF2005. We are grateful to the referee Pasquale Mazzotta for very useful comments that improved this paper. We would like to thank Rossella Cassano and Chiara Ferrari for helpful discussions. The National Radio Astronomy Observatory (NRAO) is a facility of the National Science Foundation, operated under cooperative agreement by Associated Universities, Inc. Based on photographic data obtained using The UK Schmidt Telescope. The UK Schmidt Telescope was operated by the Royal Observatory Edinburgh, with funding from the UK Science and Engineering Research Council, until 1988 June, and thereafter by the Anglo-Australian Observatory. Original plate material is copyright (c) of the Royal Observatory Edinburgh and the AngloAustralian Observatory. The plates were processed into the present compressed digital form with their permission. The Digitized Sky Survey was produced at the Space Telescope Science Institute under US Government grant NAG W-2166. This research has made use of the NASA/IPAC Extragalactic Data Base (NED) which is operated by the JPL, California Institute of Technology, under contract with the National Aeronautics and Space Administration.

\section{Appendix A: Serendipitous detection of a giant radio galaxy south-west of $\mathrm{A} 1068$}

The left panel of Fig. A.1 shows a large field of view of the radio emission around A1068 overlaid on the archive XMM observation. The XMM image is in the $0.2-5.0 \mathrm{keV}$ band and has been convolved with a Gaussian of $\sigma=20^{\prime \prime}$. The image shows a sky region south-west of A1068. The cluster central emission is located in the top left corner of the figure.

At a projected distance of about 17' from A1068, we detected an extraordinarily extended radio galaxy, which does not belong to the cluster, showing a wide-angle head-tail morphology. The core of the head-tail radio galaxy is located at $\mathrm{RA}=10^{\mathrm{h}} 39^{\mathrm{m}} 30^{\mathrm{s}} \mathrm{Dec}=39^{\circ} 47^{\prime} 19^{\prime \prime}$ and is coincident with a bright galaxy at a redshift $z=0.0929$ (taken from the Sloan 

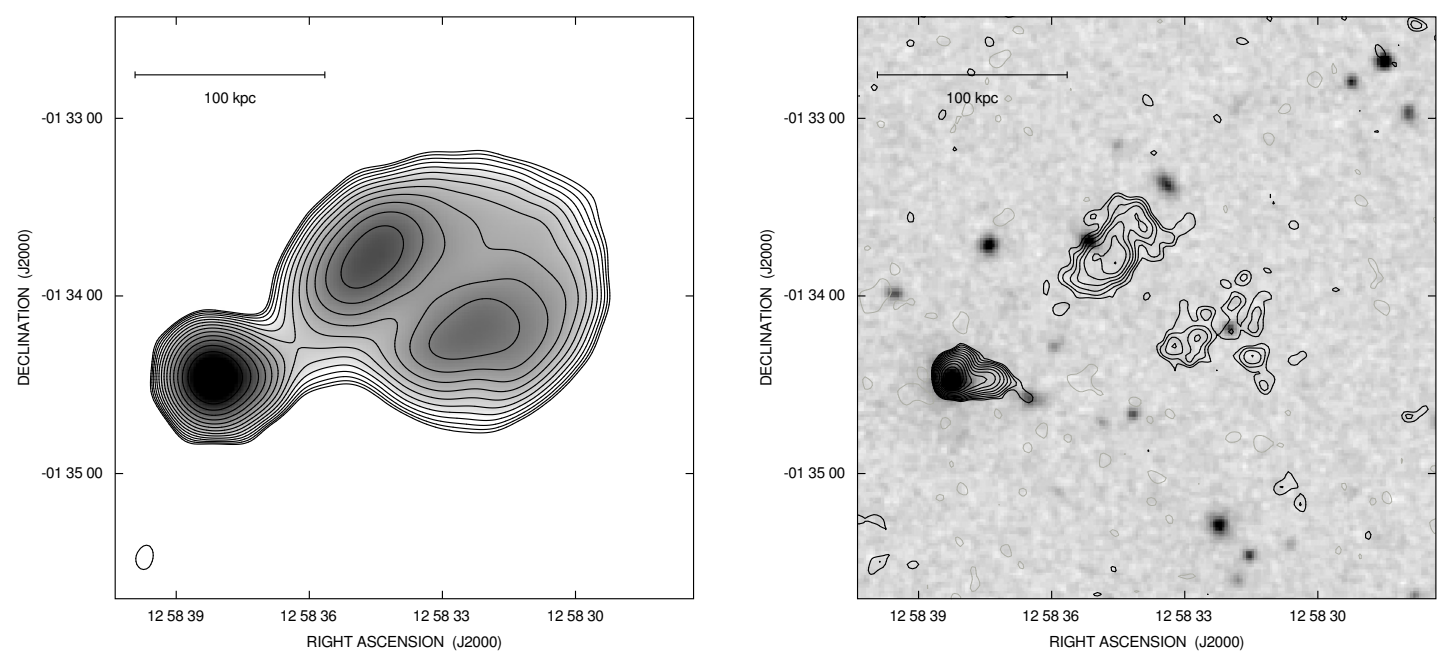

Fig. B.1. Left: total intensity radio contours to the north-west of the A1650 cluster, at $1.4 \mathrm{GHz}$ with a $F W H M$ of $16^{\prime \prime} \times 16^{\prime \prime}$. The first contour level is drawn at $0.13 \mathrm{mJy} / \mathrm{beam}$ and the rest are spaced by a factor $\sqrt{2}$. The sensitivity $(1 \sigma)$ is $0.045 \mathrm{mJy} / \mathrm{beam}$. Right: total intensity radio contours to the north-west of the A1650 cluster, at $1.4 \mathrm{GHz}$ with a FWHM of 5.4" $\times 5.4^{\prime \prime}$ taken from the FIRST survey. The bottom two contour levels are drawn at -0.3 and $0.3 \mathrm{mJy} /$ beam and the rest are spaced by a factor $\sqrt{2}$. The sensitivity of the FIRST survey $(1 \sigma)$ is $0.15 \mathrm{mJy} / \mathrm{beam}$. The contours of the radio intensity are overlaid on the optical POSS2 image.

Survey). At the distance of the head tail radio galaxy, 1" corresponds to $1.71 \mathrm{kpc}$. Therefore, its total extension reaches about $1.6 \mathrm{Mpc}$ in size. The radio emission shows a clearly unresolved core, two jets with some bright knots, and fading extended lobes. The southern jet is brighter than the northern one. Moreover, it presents a well collimated shape that bends sharply to the east at about $3^{\prime}(\simeq 300 \mathrm{kpc}$ ) from the core. The morphology of the northern jet is not as clear since it is only partially visible, although it seems to bend more gradually than the southern jet. The difference in brightness and shape of the two jets may indicate that the radio source appears shortened by projection effects. On the north side, we see a diffuse low brightness lobe, while the southern lobe is only partially detected. Because of its large extension, we may be missing flux from the entire source, and the flux density of the radio galaxy calculated in our image must therefore be considered as a lower limit. Its total flux density is $>72 \mathrm{mJy}$, which corresponds to a power $L_{1.4 \mathrm{GHz}}>1.5 \times 10^{24} \mathrm{~W} / \mathrm{Hz}$. From the NVSS, we estimated a flux density of $75 \pm 2 \mathrm{mJy}$.

In the radio/X-ray overlay, we note an extended X-ray source, which may be a small cluster or a group hosting this giant radio galaxy. The presence of a cluster is confirmed in the optical band, since it belongs to the large new catalog of galaxy clusters (Koester et al. 2007) extracted from the Sloan Digital Sky Survey optical imaging data (York et al. 2000). The interaction of the radio plasma with the thermal intracluster medium may explain its distorted morphology.

To identify the optical counterpart of the giant radio source, the right panel of Fig. A.1 shows a zoomed image of the central part of the radio galaxy overlaid on the optical image taken from the POSS2 red plate. The identification seems unambiguous.

\section{Appendix B: Serendipitous detection of an unusual radio galaxy in A1650}

Coincident with the galaxy 2MASX J12583829-0134290 $(z=0.086217)$ that is at the position $\mathrm{RA}=12^{\mathrm{h}} 58^{\mathrm{m}} 38.2^{\mathrm{s}}$ Dec $=-01^{\circ} 34^{\prime} 29^{\prime \prime}$, we detected an unusual radio galaxy. This radio galaxy was also observed by Owen et al. (1993) in a $1.4 \mathrm{GHz}$
VLA survey of Abell clusters of galaxies. The left panel of Fig. B.1 shows the radio emission with a resolution of $16^{\prime \prime}$. The morphology of the source appears to be a core with, to the northwest, a round structure with sharp edges. The flux density of the overall structure is $135 \pm 4 \mathrm{mJy}$. The right panel of Fig. B. 1 shows the image taken from the FIRST survey overlaid on the POSS2 image. The higher resolution image shows that the round structure we detected at lower resolution is not due to the radio emission from another galaxy. It may be a NAT (narrow-angle tailed) source. The faint extended emission visible at higher resolution could be the ends of the two tails. A similar source was detected in A3921 (Ferrari et al. 2006), a NAT source associated with a galaxy, whose diffuse component is a partly detached pair of tails from an earlier period of activity of the galaxy.

The Chandra field of view does not extend to the radio galaxy. We retrieved the larger field of view XMM observation from the archive but no diffuse X-ray emission was detected at the location of the radio galaxy.

\section{References}

Ascasibar, Y., \& Markevitch, M. 2006, ApJ, 650, 102

Bacchi, M., Feretti, L., Giovannini, G., \& Govoni, F. 2003, A\&A, 400, 465

Baldi, A., Ettori, S., Mazzotta, P., et al. 2007, ApJ, 666, 835

Becker, R. H., White, R. L., \& Helfand, D. J. 1995, ApJ, 450, 559

Best, P. N., von der Linden, A., Kauffmann, G., Heckman, T. M., \& Kaiser, C. R. 2007, MNRAS, 379, 894

Bîrzan, L., McNamara, B. R., Nulsen, P. E. J., et al. 2008, ApJ, 686, 859

Bourdin, H., \& Mazzotta, P. 2008, A\&A, 479, 307

Brunetti, G., Setti, G., Feretti, L., \& Giovannini, G. 2001, MNRAS, 320, 365

Buote, D. A. 2001, ApJ, 553, L15

Buote, D. A., \& Tsai, J. C. 1995, ApJ, 452, 522

Buote, D. A., \& Canizares, C. R. 1996, ApJ, 457, 565

Burns, J. O. 1990, AJ, 99, 14

Burns, J. O., Sulkanen, M. E., Gisler, G. R., \& Perley, R. A. 1992, ApJ, 388, L49 Cassano, R., Gitti, M., \& Brunetti, G. 2008, A\&A, 486, L31

Clarke, T. E., Blanton, E. L., \& Sarazin, C. L. 2004, ApJ, 616, 178

Condon, J. J., Cotton, W. D., Greisen, E. W., et al. 1998, AJ, 115, 1693

Dolag, K., \& Enßlin, T. A. 2000, A\&A, 362, 151

Donahue, M., Voit, G. M., O’Dea, C. P., Baum, S. A., \& Sparks, W. B. 2005, ApJ, 630, L13 
Dressler, A. 1978, ApJ, 223, 765

Dunn, R. H., Fabian, A. C., \& Taylor, G. B. 2005, MNRAS, 364, 1343

Eckert, D., Produit, N., Paltani, et al. 2008, A\&A, 479, 27

Fabian, A. C. 1994, ARA\&A, 32, 277

Feretti, L., \& Giovannini, G. 2008, A Pan-Chromatic View of Clusters of Galaxies and the Large-Scale Structure, 740, 143

Ferrari, C., Hunstead, R. W., Feretti, L., Maurogordato, S., \& Schindler, S. 2006, A\&A, 457, 21

Ferrari, C., Govoni, F., Schindler, S., Bykov, A. M., \& Rephaeli, Y. 2008, Space Sci. Rev., 134, 93

Fujita, Y., Kohri, K., Yamazaki, R., \& Kino, M. 2007, ApJ, 663, L61

Fujita, Y., Hayashida, K., Nagai, M., et al. 2008, PASJ, 60, 1133

Giovannini, G., \& Feretti, L. 2000, New Astron., 5, 335

Giovannini, G., Tordi, M., \& Feretti, L. 1999, New Astron., 4, 141

Gitti, M., Brunetti, G., \& Setti, G. 2002, A\&A, 386, 456

Gitti, M., Brunetti, G., Feretti, L., \& Setti, G. 2004, A\&A, 417, 1

Gitti, M., Ferrari, C., Domainko, W., Feretti, L., \& Schindler, S. 2007, A\&A, 470, L25

Govoni, F., Feretti, L., Giovannini, G., et al. 2001, A\&A, 376, 803

Govoni, F., Markevitch, M., Vikhlinin, A., et al. 2004, ApJ, 605, 695

Kempner, J. C., \& Sarazin, C. L. 2001, ApJ, 548, 639

Koester, B. P., McKay, T. A., Annis, J., et al. 2007, ApJ, 660, 239

Lewis, A. D., Stocke, J. T., \& Buote, D. A. 2002, ApJ, 573, L13

Majerowicz, S., Neumann, D. M., \& Reiprich, T. H. 2002, A\&A, 394, 77

Markevitch, M. 2002 [arXiv:astro-ph/0205333]

Markevitch, M., Vikhlinin, A., \& Forman, W. R. 2003, ASP Conf. Ser., 301, 37
Marković, T., Owen, F. N., \& Eilek, J. A. 2004, The Riddle of Cooling Flows in Galaxies and Clusters of galaxies, 61

Mazzotta, P., \& Giacintucci, S. 2008, ApJ, 675, L9

McNamara, B. R., \& Nulsen, P. E. J. 2007, ARA\&A, 45, 117

McNamara, B. R., Wise, M. W., \& Murray, S. S. 2004, ApJ, 601, 173

Owen, F. N., White, R. A., \& Ge, J. 1993, ApJS, 87, 135

Pedlar, A., Ghataure, H. S., Davies, R. D., et al. 1990, MNRAS, 246, 477

Peterson, J. R., Paerels, F. B., Kaastra, J. S., et al. 2001, A\&A, 365, L104

Petrosian, V. 2001, ApJ, 557, 560

Petrosian, V., Bykov, A., \& Rephaeli, Y. 2008, Space Sci. Rev., 134, 191

Pfrommer, C., \& Enßlin, T. A. 2004, A\&A, 413, 17

Pfrommer, C., Enßlin, T. A., \& Springel, V. 2008, MNRAS, 385, 1211

Pratt, G.W., \& Arnaud, M. 2002, A\&A, 394, 375

Rephaeli, Y., Nevalainen, J., Ohashi, T., \& Bykov, A. M. 2008, Space Sci. Rev., 134, 71

Rengelink, R. B., Tang, Y., de Bruyn, A. G., et al. 1997, A\&AS, 124, 259

Sarazin, C. L., Wise, M. W., \& Markevitch, M. L. 1998, ApJ, 498, 606

Schmidt, R. W., Allen, S. W., \& Fabian, A. C. 2001, MNRAS, 327, 1057

Takahashi, S., \& Yamashita, K. 2003, PASJ, 55, 1105

Taylor, G. B., Barton, E. J., \& Ge, J. 1994, AJ, 107, 1942

Uson, J. M., Boughn, S. P., \& Kuhn, J. R. 1991, ApJ, 369, 46

Wise, M. W., McNamara, B. R., \& Murray, S. S. 2004, ApJ, 601, 184

York, D. G., Adelman, J., Anderson, J. E., et al., 2000, AJ, 120, 1579

Venturi, T., Giacintucci, S., Brunetti, G., et al. 2007, A\&A, 463, 937

Venturi, T., Giacintucci, S., Dallacasa, D., et al. 2008, A\&A, 484, 327

Vikhlinin, A., Markevitch, M., Murray, S. S., et al. 2005, ApJ, 628, 655 